\title{
ON THE STRUCTURE OF SEMIPRIME RINGS
}

\author{
AUGUSTO H. ORTIZ
}

ABSTRACT. The structure of prime rings has recently been studied by A. W. Goldie, R. E. Johnson, L. Lesieur and R. Croisot. In their main results some sort of finiteness assumption is invariably made. It is shown in this paper that certain semiprime rings are subdirect sums of full rings of linear transformations of a right vector space over a division ring. No finiteness assumption is made about the ring. An apparently new radical property is defined and some of its properties are established; e.g., the radical of a matrix ring $R_{n}$ is the matrix ring of the radical of $R$.

1. Introduction. Our results were motivated by the following characterization of primitive ideals in terms of prime ideals. Throughout, if $M$ is an $R$-module and $N$ is a submodule of $M,(0: M)=\{a \in R \mid M a=0\}$ and $(N: M)=\{a \in R \mid M a \subset N\}$.

THEOREM 1.1. A prime ideal $P$ of a ring $R$ is primitive if and only if there exists an $R$-module $M$ such that $(0: M)=P$ which satisfies the following two conditions:

(1) If $N$ is a submodule of $M$ such that $(0: N)=(0: M)$, then $N=M$.

(2) If $N$ is a submodule of $M$ such that $(N: M)=(0: M)$, then $N=0$.

The sufficiency of the conditions is easy to establish since $(N: M)(0: N) \subset$ $(0: M)$ and $(0: M)$ is a prime ideal of $R$. Whence, using (1) and (2), $N=0$ or $N=M$. The necessity is more evident. There are trivial examples of modules which satisfy (1) and (2) but which are not irreducible. Also there are examples of $R$-modules $M$ such that $(0: M)$ is a prime ideal of $R$ but $M$ does not satisfy condition (2). This theorem suggests conditions which may be imposed on a prime ring to obtain information about its structure. In effect, we prove in Theorem 3.3 that if $R$ is a prime ring for which there is a module $M$ such that $(0: M)=0$ and satisfying condition

Received by the editors June 19, 1972.

AMS (MOS) subject classifications (1970). Primary 16A12, 16A21, 16A48; Secondary 16A20.

Key words and phrases. Finiteness assumption, subdirect sums, radical property, primitive ideals, prime ideals, irreducible module, the prime radical, the Jacobson radical, hereditary radical, Prüfer module, essential submodule, small submodule, subdirectly irreducible, heart of a module, injective hull, quasi-injective hull, essential extension. 
(2) above, then $R$ may be embedded in a full ring of linear transformations of a right vector space over a division ring.

2. Radical property $K$. Let $K_{R}$ denote the class of all $R$-modules $M$ such that $(0: M)$ is a prime ideal of $R$ and whose only submodule $N$ for which $(N: M)=(0: M)$ is $N=0$. We say that $K_{R}$ is faithful if

$$
\bigcap\left\{(0: M) \mid M \in K_{R}\right\}=0 \text {. }
$$

THEOREM 2.1. The property $K_{R}=\varnothing$ is a radical property of $R$.

Proof. It suffices to show [1] that for every $R$-module $M$ and every homomorphism $h$ of $R$ the following two conditions are satisfied:

(1) $M \in K_{R h}$ if and only if $M \in K_{R}$ and $\operatorname{Ker} h \subset(0: M)$.

(2) $K_{R}$ is faithful if and only if for every ideal $I \neq 0$ of $R, K_{I} \neq \varnothing$.

It is easy to check property (1). To see that (2) holds, suppose that $K$ is faithful and that $I \neq 0$ is an ideal of $R$. Then there exists $M \in K_{R}$ such that $M I \neq 0$. Whence $(0: M)_{I}=(0: M)_{R} \cap I$ is a prime ideal of $I$. Furthermore if $N$ is an $I$-submodule of $M$ such that $(N: M)_{I}=(0: M)_{I}$, then $N \subset\{x \in M \mid x I \subset N\} \equiv N^{\prime}$, an $R$-submodule of $M$. Also $\left(N^{\prime}: M\right)_{R}=$ $(0: M)_{R}$, so that $N^{\prime}=0$ and $N=0$. Therefore $M \in K_{I}$.

Conversely, if for every ideal $I \neq 0$ of $R, K_{I} \neq \varnothing$, and if

$$
J=\bigcap\left\{(0: M) \mid M \in K_{R}\right\} \neq 0,
$$

then $K_{J} \neq \varnothing$. If $M \in K_{J}$, it is not hard to show that $M J \in K_{R}$. But $J \notin$ $(0: M J)_{R}$ and this is a contradiction. Therefore $J$ must be 0 and the proof is complete.

We shall denote by $K$ the radical property defined by the classes $K_{R}$. It is easy to show that, for each ring $R, K(R)=\bigcap\left\{(0: M) \mid M \in K_{R}\right\}$. In view of Theorem 1.1, $K$ contains the prime radical $P$ and is contained in the Jacobson radical $J$. We shall give examples which show that $K \neq J$, but we do not know yet that $K \neq P$.

It follows from the last paragraph of the preceding theorem that $K_{R} \neq \varnothing$ when $K_{I} \neq \varnothing$, where $I$ is an ideal of $R$. Therefore $K$ is hereditary.

The fact that the ring of integers is $K$-semisimple implies [4, Theorem 2, p. 11] that $K\left(R^{1}\right)=K(R)$, where $R^{1}$ denotes the ring with identity in which $R$ is usually embedded as an ideal. This fact simplifies the proof of the next theorem. In what follows $R_{n}$ denotes the ring of $n \times n$ matrices over $R$. We will say that an ideal $P$ of $R$ is $K$-primitive if $P=(0: M)$ for some $M \in K_{R}$. A ring $R$ will be called $K$-primitive if 0 is a $K$-primitive ideal of $R$. It is clear that every primitive ideal is . rimitive and that each $K$ primitive ideal is prime.

THEOREM 2.2. $K\left(R_{n}\right)=K(R)_{n}$. 
Proof. We suppose that $R$ contains an identity. It suffices to show that $R$ is $K$-primitive if and only if $R_{n}$ is $K$-primitive. If $R$ is $K$-primitive, then there is $M \in K_{R}$ such that $(0: M)=0$. Let $M^{\prime}$ be the $R_{n}$-module of $1 \times n$ matrices over $M$. It is easy to see that $\left(0: M^{\prime}\right)=0$ which is a prime ideal of $R_{n}$ [5, Theorem 4.28, p. 72]. If $N^{\prime}$ is an $R_{n}$-submodule of $M^{\prime}$ such that $\left(N^{\prime}: M^{\prime}\right)=0$, then the set $N$ of all the entries of the elements of $N^{\prime}$ is an $R$-submodule of $M$ and also $(N: M)=0$. Therefore $N=0$ and $N^{\prime}=0$, so that $M^{\prime} \in K_{R_{n}}$ and $R_{n}$ is $K$-primitive.

Conversely, if $R_{n}$ is $K$-primitive, then there is $M^{\prime} \in K_{R_{n}}$ such that $\left(0: M^{\prime}\right)=0$. Let $M=\left\{x E_{i 1} \mid x \in M^{\prime}, 1 \leqq i \leqq n\right\}$, where $E_{i 1}$ denotes the $n \times n$ matrix with 1 in the $(i, 1)$ position and 0 elsewhere. Then $M$ is an $R$ module such that $(0: M)=0$ which again is a prime ideal of $R$. If $N$ is an $R$-submodule of $M$ such that $(N: M)=0$, then $N^{\prime}=\left\{\sum x E_{1 j} \mid x \in N\right.$, $1 \leqq j \leqq n\}$ is an $R_{n}$-submodule of $M^{\prime}$ such that $\left(N^{\prime}: M^{\prime}\right)=0$. For if $A \in$ $\left(N^{\prime}: M^{\prime}\right)$, say $A=\left(a_{i j}\right)$, and if $y \in M$, say $y=x E_{p 1}$, where $x \in M^{\prime}$, then for each $i, j y a_{i j}=x E_{p 1} a_{i j}=x E_{p i} A E_{j 1}=z E_{j 1}$, where $z \in N^{\prime}$. Let $z=$ $\sum w E_{1 k}$, where each $w \in N$. Then $z E_{j 1}$ is an element of the form $w E_{11}=w$ in $N$ if some of the $k$ 's happen to be $j$, or $z E_{j 1}$ is 0 if none of the $k$ 's is $j$. In any case $z E_{j 1} \in N$ so that $y a_{i j} \in N$ for every $y \in M$. Thus $a_{i j} \in(N: M)=0$ for all $i, j$, and $A=0$. Therefore $\left(N^{\prime}: M^{\prime}\right)=0$. Since $M^{\prime} \in K_{R_{n}}, N^{\prime}=0$. This implies that $N=0$, for if $y \in N$, then $y=y E_{11} \in N^{\prime}=0$. The proof is now complete.

All the previous results are valid also for the class $H_{R}$ of all $R$-modules $M$ such that $(0: M)$ is a prime ideal of $R$ and whose only submodule $N$ such that $(0: N)=(0: M)$ is $N=M$. The proofs are analogous. Moreover the radical property $H$ is inherited by right ideals.

If $D$ is an integral domain (not necessarily with identity), then $D \in K_{D}$ and $(0: D)=0$ so that $D$ is $K$-primitive. (This fact was first observed by Mr. Rafael Cardona Oviedo.) Hence $K \neq J$, the Jacobson radical. In fact, the commutative $K$-primitive rings are exactly the integral domains. Hence for commutative rings, $K(R)=P(R)$, the prime radical. The arbitrary $K$-primitive rings are studied in the next section.

An example showing that $H \neq J$ is any nonsemisimple principal ideal domain since these are $H$-semisimple. Let $R$ be a principal ideal domain with identity and let $p$ be a generator of a maximal ideal of $R$. Then $\cap p^{i} R=0$. Take as a unital $R$-module $M$, the general Prüfer module $R\left(p^{\infty}\right)$ (cf. [3, p. 15] or [2, p. 143]) generated by $x_{1}, x_{2}, \cdots, x_{n}, \cdots$ such that $x_{1} p=0, x_{2} p=x_{1}, \cdots, x_{n+1} p=x_{n}, \cdots$. Then the only proper submodules of $M$ are $x_{1} R<x_{2} R<\cdots<x_{n} R<\cdots$. For each of these, $\left(0: x_{i} R\right)=p^{i} R$, while $(0: M)=0$. Therefore $M \in H_{R}$ and $R$ is $H$-primitive.

3. $K$-primitive rings. If $M \in K_{R}$, then $M$ is uniform in the sense that every nonzero submodule is essential. This is easy to see since if $N$ and $N^{\prime}$ 
are submodules of $M$ such that $N \cap N^{\prime}=0$, then $(N: M) \cap\left(N^{\prime}: M\right)=$ $\left(N \cap N^{\prime}: M\right)=(0: M)$ so that $(N: M)=(0: M)$ or $\left(N^{\prime}: M\right)=(0: M)$ and $N=0$ or $N^{\prime}=0$. Moreover the only submodules of $M$ of the form $I^{*}=$ $\{x \in M \mid x I=0\}$, where $I$ is an ideal of $R$, are 0 and $M$. (Dually, if $M \in H_{R}$, then every proper submodule of $M$ is small. Also the only submodules of $M$ of the form $M I$, where $I$ is a right ideal of $R$, are 0 and $M$.)

LEMMA. If $R$ is a subdirectly irreducible $K$-primitive ring, say $0=(0: M)$, where $M \in K_{R}$, then $M$ is subdirectly irreducible.

Proof. Let $N_{i}$ be submodules of $M$ such that $\cap N_{i}=0$. Then $\bigcap\left(N_{i}: M\right)=\left(\bigcap N_{i}: M\right)=0$. Since $R$ is subdirectly irreducible, $\left(N_{i}: M\right)=0$ for some $i$. Hence some $N_{i}=0$.

THEOREM 3.1. Every K-primitive subdirectly irreducible ring is primitive.

Proof. If $R$ is a $K$-primitive ring, then there is $M$ in $K_{R}$ such that $(0: M)=0$. Let $V$ be the heart of $M$. Then $V$ is an irreducible $R$-module and $(0: V)=0$ since $V \neq 0$ implies $(V: M) \neq(0: M)$ so that $(0: V)=$ $(0: M)=0$.

Therefore, for subdirectly irreducible rings $R, K(R)=J(R)$. In what follows, $\hat{M}$ and $\bar{M}$ denote the injective hull and the quasi-injective hull of $M$, respectively. We follow the ideas and result of [6].

THEOREM 3.2. If $M \in K_{R}$, then $D=\operatorname{Hom}_{R}(\bar{M}, \bar{M})$ is a division ring.

Proof. Let $k \in \operatorname{Hom}_{R}(\hat{M}, \hat{M})$ such that $M k \neq 0$. Since $\hat{M}$ is an essential extension of $M, N=M k \cap M \neq 0$. Then $(0: N)=(0: M)$ because $M \in K_{R}$. But $(\operatorname{Ker} k \cap M: M) \subset(0: N)$ so that $\operatorname{Ker} k \cap M=0$ and $\operatorname{Ker} k=0$. Applying the lemma in [6], $D$ is a division ring.

It follows now from the results of [6] that if $M \in K_{R}$ and $x, y \in M$, then $(0: x)>(0: y)$ implies $x=0$. If $N$ is a nonzero submodule of $M$, then $(N: x)=(0: y)$ implies $y=0$.

THEOREM 3.3. Every K-primitive ring is a subring of a full ring of linear transformations of a vector space over a division ring.

Proof. Let $M \in K_{R}$ such that $(0: M)=0$. Then $M$ is a right vector space over $D=\operatorname{Hom}_{R}(\bar{M}, \bar{M})$. For each $a \in R$, let $a^{\prime} \in \operatorname{Hom}_{D}(M, M)$ defined by $x a^{\prime}=x a$ for all $x \in M$. The map $a \rightarrow a^{\prime}$ is a ring monomorphism of $R$ into $\operatorname{Hom}_{D}(M, M)$.

COROLlary. Every $K$-semisimple ring is a subdirect sum of subrings of full linear rings.

ADDED IN PROOF. Any simple radical ring is prime and subdirectly irreducible, hence $K$-radical. Thus $P \neq K$. 


\section{REFERENCES}

1. V. A. Andrunakievič and Ju. M. Rjabuhin, Modules and radicals, Dokl. Akad. Nauk SSSR 156 (1964), 991-994=Soviet Math. Dokl. 5 (1964), 728-731. MR 32 \#7596.

2. T. Chi-Te, Report on injective modules, Queen's Papers in Pure and Appl. Math., no. 6, Queen's University, Kingston, Ontario, 1966.

3. L. Fuchs, Infinite abelian groups. Vol. I, Pure and Appl. Math., vol. 36, Academic Press, New York, 1970. MR 41 \#333.

4. N. Jacobson, Structure of rings, rev. ed., Amer. Math. Soc. Colloq. Publ., vol. 37, Amer. Math. Soc., Providence, R.I., 1964. MR 36 \#5158.

5. N. H. McCoy, The theory of rings, Macmillan, New York, 1964. MR 32 \#5680.

6. E. T. Wong, Endomorphims of the quasi-injective hull of a module, Canad. Math. Bull. 13 (1970), 149-150.

Department of Mathematics, University of Puerto Rico, Mayaguez, Puerto Rico 00708 